\title{
Брэгговский резонанс в системе плазмонных нановключений AsSb B AlGaAs
}

\author{
(C) В.И. Ушанов+, В.В. Чалдышев ${ }^{+}$, В.В. Преображенский*, М.А. Путято*, Б.Р. Семягин* \\ + Физико-технический институт им. А.Ф. Иоффре Российской академии наук, \\ 194021 Санкт-Петербург, Россия \\ * Институт фризики полупроводников Сибирского отделения Российской академии наук, \\ 630090 Новосибирск, Россия \\ E-mail: Decorus2009@mail.ru, chald.gvg@mail.ioffe.ru
}

(Получена 27 апреля 2016 г. Принята к печати 10 мая 2016 г.)

Исследовалось оптическое отражение от периодических структур на основе полупроводниковой матрицы $\mathrm{AlGaAs,} \mathrm{содержащей} \mathrm{двумерные} \mathrm{массивы} \mathrm{плазмонных} \mathrm{нановключений} \mathrm{AsSb.} \mathrm{Число} \mathrm{слоев} \mathrm{нановключений}$ составляло 12 и 24, номинальный период расположения слоев - 100 или 110 нм соответственно. В экспериментальных спектрах коэффициента оптического отражения при нормальном падении нами наблюдалась резонансная брэгговская дифракция с главными пиками на длинах волн 757 и 775 нм (1.64 и 1.60 эВ), в зависимости от пространственного периода наноструктуры. Амплитуды резонансных пиков достигали 22 и $31 \%$ при числе слоев 12 и 24, при том что объемная доля нановключений была существенно меньше $1 \%$. В случае наклонного падения света картина брэгговской дифракции смещалась в соответствии с законом Вульфа-Брэгга. Методом матриц переноса выполнено численное моделирование экспериментальных данных с учетом пространственной геометрии структуры и резонансных особенностей плазмонных слоев AsSb.

\section{1. Введение}

Развитие фотоники связано с разработкой новых композитных метаматериалов, чьи свойства определяются внутренней структурой и взаимодействием света с составляющими их элементами, размеры которых много меньше длины волны света. Метаматериалы могут быть получены путем формирования хаотических или упорядоченных массивов нановключений различной формы и различного состава, что дает возможность существенно модифицировать диэлектрическую и магнитную проницаемости вещества $[1,2]$. В частности, особый интерес представляет полупроводниковая матрица, содержащая массивы нановключений металлической фазы, благодаря своим уникальным электронным и оптическим свойствам, которые можно контролировать путем варьирования объемной доли металлической компоненты в процессе роста [3-5].

В случае, если размеры нановключений и расстояния между ними много меньше длины волны света, оптические свойства композитной среды можно рассматривать в рамках приближения эффективной среды. В пределе сильно разреженных дисперсий наночастиц, когда их объемная доля мала, вклад каждого нановключения в оптические свойства вещества линеен. Дисперсия эффективной диэлектрической функции метаматериала,

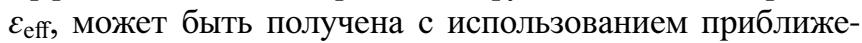
ния Максвелла-Гарнетта [6]. При этом в оптических свойствах метаматериала следует ожидать особенностей, связанных с поглощением света поверхностными плазмонами в системе металлических нановключений в полупроводниковой среде, при выполнении условия

$$
\operatorname{Re}\left(\varepsilon_{m}+2 \varepsilon_{s}\right)=0,
$$

где $\varepsilon_{s}$ и $\varepsilon_{m}-$ диэлектрические функции полупроводниковой и металлической компонент соответственно.
Если расстояние между наночастицами ставится сравнимым с длиной волны света, проходящего через структуру, приближение эффективной среды становится неприменимым [7]. Периодичность в расположении наночастиц должна вызывать брэгговскую дифракцию света с главным резонансным пиком на длине волны, определяемой из закона Вульфа-Брэгга

$$
\lambda_{\mathrm{Br}}=2 d \sqrt{n_{\mathrm{eff}}^{2}-\sin ^{2} \theta},
$$

где $d-$ пространственный период структуры метаматериала, $n_{\mathrm{eff}}$ - эффективный показатель преломления, $\theta$ - угол падения света. В области брэгговского резонанса взаимодействие света с метаматериалом оказывается усиленным.

В последнее время большой интерес сосредоточен на метаматериалах, представляющих собой разреженные массивы наночастиц металла в полупроводниковой матрице GaAs или AlGaAs высокого кристаллического качества. Отчасти его возникновению способствовала разработка технологии выращивания GaAs при низкой температуре методом молекулярно-лучевой эпитаксии (МЛЭ). В процессе МЛЭ при низкой температуре в матрицу GaAs захватывается избыточный As в концентрации от 1 до 2\%, формируя метастабильную систему точечных дефектов $\mathrm{As}_{\mathrm{Ga}}$ в сильно нестехиометрическом GaAs $[8,9]$. Последующий отжиг приводит к самоорганизации неупорядоченной системы нановключений металлического As в объеме эпитаксиального слоя. Формирование периодической системы слоев нановключений $\mathrm{AsSb}$ может быть достигнуто в процессе $\delta$-легирования эпитаксиального слоя изовалентной примесью $\mathrm{Sb}[5,10]$. Размер и плотность металлических нановключений могут быть заданы путем установления необходимых температуры роста, температуры и продолжительности отжига, что создает основу для разработки приборов на 
Параметры образцов

\begin{tabular}{c|c|c|c|c|c}
\hline Образцы & $\begin{array}{c}\text { Материал } \\
\text { матрицы }\end{array}$ & $\begin{array}{c}\text { Период } \\
\text { структуры, нм }\end{array}$ & $\begin{array}{c}\text { Число } \\
\text { периодов }\end{array}$ & $\begin{array}{c}\text { Энергия брэгговского } \\
\text { (плазмонного) резонанса, эВ }\end{array}$ & $\begin{array}{c}\text { Амплитуда брэгговского } \\
\text { резонанса, \% }\end{array}$ \\
\hline ВP2444 & $\mathrm{AlGaAs}$ & 100 & 12 & 1.64 & $7-22$ \\
$\mathrm{ST} 1279$ & $\mathrm{AlGaAs}$ & 110 & 24 & 1.60 & $23-31$ \\
{$[13,14]$} & $\mathrm{AlGaAs}$ & Апериодическая & - & $(1.48)$ & - \\
{$[15]$} & $\mathrm{GaAs}$ & 200 & 7 & 0.86 & 22 \\
{$[16]$} & $\mathrm{AlGaAs}$ & 100 & 12 & 1.65 & -
\end{tabular}

основе полупроводниковых структур GaAs, содержащих металлические нановключения $[4,11]$.

Резонансное оптическое поглощение в неупорядоченной системе нановключений AsSb в AlGaAs впервые было обнаружено и исследовано в работах $[12,13]$. Энергия плазмонного резонанса оказалась равной $(1.47 \pm 0.01)$ эВ. В ранней работе [14] была создана периодическая структура двумерных массивов нановключений AsSb в матрице GaAs с брэгговским резонансом на длине волны 1450 нм $(0.86$ эВ). Вследствие существенной разницы частот брэгговского и плазмонного резонансов амплитуда пика оптического отражения была мала. Существенно больший резонансный пик оптического отражения был получен в работе [15], когда энергии брэгговского и плазмонного резонансов были близки.

Цель настоящей работы заключалась в создании брэгговских структур, содержащих 12 и 24 периода двумерных массивов нановключений $\mathrm{AsSb}$ в матрице $\mathrm{AlGaAs}$ и экспериментальном исследовании и численном моделировании резонансного оптического отражения при различных углах падения и поляризациях света.

\section{2. Образцы и методика эксперимента}

Метаматериалы были выращены методом МЛЭ при низкой температуре $\left(200^{\circ} \mathrm{C}\right)$ на подложке полуизолирующего GaAs c ориентацией (001) и представляли собой твердый раствор AlGaAs, периодически $\delta$-легированный изовалентной примесью $\mathrm{Sb}$. Толщина $\delta$-слоев была 1 монослой. Система $\delta$-слоев служила прекурсором для формирования двумерных массивов нановключений AsSb в процессе последующего высокотемпературного отжига. B области полупроводниковой матрицы AlGaAs между $\delta$-слоями происходило формирование неупорядоченной системы нановключений As. Средний размер нановключений увеличивался с ростом температуры отжига. Толщину слоя $\mathrm{AsSb}$ можно оценить в два характерных размера нановключений AsSb. Номинальная концентрация AlAs составляла 30\%, что обеспечивало окно прозрачности для света с длиной волны 700 нм и более $(1.75$ эВ и менее). Вследствие технологии роста образцов, аналогичной таковой для структур из работ $[12,13]$, объемную долю нановключений $\mathrm{AsSb}$ после отжига можно оценить как «1\%. Были выращены два образца с различным числом периодов.
Образец ВР2444 представлял собой эпитаксиальный слой $\mathrm{Al}_{0.3} \mathrm{Ga}_{0.7} \mathrm{As}$ толщиной 1.3 мкм, в котором в процессе роста была сформирована периодическая система из $12 \delta$-слоев $\mathrm{Sb}$ с номинальным периодом расположения 100 нм. Образец был разделен на 5 частей, 4 из которых были подвергнуты отжигу при температурах $400-700^{\circ} \mathrm{C}$.

Образец ST1279 представлял собой твердый раствор $\mathrm{Al}_{0.3} \mathrm{Ga}_{0.7} \mathrm{As}$ толщиной 2.6 мкм, содержащий упорядоченную систему из $24 \delta$-слоев $\mathrm{Sb}$ с периодом расположения 110 нм. Образец был разделен на 2 части, которые были подвергнуты отжигу при температурах 600 и $700^{\circ} \mathrm{C}$.

Параметры образцов, исследованных в данной работе и ранее в работах [12-15], представлены в таблице.

Измерения коэффициента оптического отражения осуществлялись при нормальном падении, а также при падении света под углами от 7.5 до $85^{\circ}$ для $s$ - и $p$-поляризаций в диапазоне длин волн $600-1000 \mathrm{Hм}$ при комнатной температуре. Источником света служила галогеновая лампа Osram HLX 100W 6.6 с коллиматором. Для передачи света от источника к образу и от образца к детектору использовались оптоволоконные кабели фирмы Ocean Optics. Для поляризации света использовалась призма Глана-Тейлора. Запись спектров осуществлялась при помощи спектрометра Ocean Optics QE65Pro и программного обеспечения Ocean Optics SpectraSuite software.

\section{3. Результаты эксперимента}

Спектры коэффициента оптического отражения при нормальном падении для образцов ВР2444 и ST1279 представлены на рис. 1. Видно, что в спектрах обоих образцов наблюдается пик резонансного отражения, сопровождающийся сателлитными осцилляциями. Для неотожженной (as-grown) части образца ВР2444 значительных резонансных особенностей в отражении света от системы $\delta$-слоев $\mathrm{Sb}$ не наблюдается. Амплитуды пиков резонансного отражения увеличиваются с ростом температуры отжига, т. е. с возникновением и увеличением размеров наночастиц AsSb. Для образца BP2444 амплитуды пиков составили 7\% для неотожженной части образца, $14 \%$ для температуры отжига $400^{\circ} \mathrm{C}, 20 \%$ для $500^{\circ} \mathrm{C}, 22 \%$ для $600^{\circ} \mathrm{C}$ и $19 \%$ для $700^{\circ} \mathrm{C}$. Для образца ST1279 величины резонансных амплитуд составили 23\% для температуры отжига $600^{\circ} \mathrm{C}$ и $31 \%$ для $700^{\circ} \mathrm{C}$. 


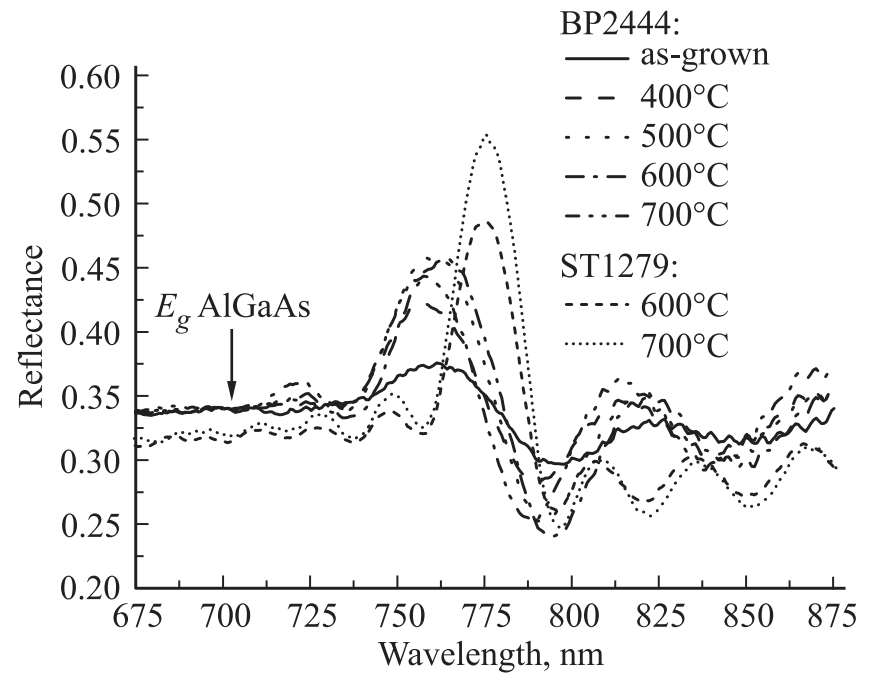

Рис. 1. Экспериментальные зависимости коэффициента оптического отражения при нормальном падении для образцов ВР2444 и ST1279.

Резонансные длины волн соответствовали ожидаемым значениям в соответствии с законом Вульфа-Брэгга. Для образца ВР2444 длина волны резонансного отражения равнялась $757 \mathrm{HM}$ (1.64 эB), для ST1279 775 нм (1.60 эВ). Полные ширины на половине высоты для частей BP2444 и ST1279, отожженных при температуре $700^{\circ} \mathrm{C}$, составили $44 \mathrm{Hм}(0.11$ эВ $)$ и $28 \mathrm{Hм}$ $(0.06$ эВ) соответственно. Период боковых осцилляций для образца ST1279 был в 2 раза меньше такового для BP2444 вследствие вдвое большего числа слоев AsSb в ST1279. Стрелкой на рисунке отмечены особенности, связанные с краем фундаментального поглощения в матрице AlGaAs.

\section{4. Обсуждение}

Резонансная энергия пика брэгговской дифракции определяется величиной пространственного периода структуры и в работе [14] составляла 0.86 эВ. Энергия плазмонного резонанса в то время известна не была. Параметры плазмонного резонанса в неупорядоченной системе $\mathrm{AsSb}-\mathrm{AlGaAs}$ были определены нами в работах $[12,13]$. Оказалось, что энергия резонанса составляет $(1.47 \pm 0.01)$ эВ при ширине $(0.19 \pm 0.01)$ эВ и практически не зависит от размера наночастиц $\mathrm{AsSb}$ в исследованном диапазоне средних размеров 6-8нм. Таким образом, вероятной причиной малой амплитуды резонансного отражения света периодической системой наночастиц AsSb в работе [14] является существенная разность частот брэгговского и плазмонного резонансов.

Сравнение экспериментальных данных из работы [14] c результатами настоящего исследования позволяет предположить, что в случае сближения частот брэгговского и плазмонного резонансов нами наблюдается усиление взаимодействия света с системой нановключений AsSb в AlGaAs. В области плазмонного резонанса диэлектрическая проницаемость нановключений $\mathrm{AsSb}$ отрицательна, и, чем ближе к нему находится частота излучения, тем больше становится отрицательная проницаемость. Диэлектрическая проницаемость полупроводниковой матрицы $\mathrm{AlGaAs}$ положительна во всем исследуемом диапазоне. Наблюдаемая в данном исследовании картина интенсивной брэгговской дифракции связана с высоким диэлектрическим контрастом между полупроводниковой матрицей и нановключениями металлической фазы. Энергию плазмонного резонанса для периодической системы нановключений AsSb в AlGaAs, исследуемой в данной работе, мы также полагаем равной соответствующей энергии для неупорядоченной системы $\mathrm{AsSb}-\mathrm{AlGaAs}$ из работ $[12,13]$

Следует отметить, что в контрольном образце, представлявшем собой эпитаксиальный слой $\mathrm{AlGaAs}$ высокого кристаллического качества и не содержащем нановключений, а также в образце, содержащем неупорядоченную систему нановключений $\mathrm{AsSb}$, резонансных особенностей в оптических спектрах отражения обнаружено не было [15]. Исследование экспериментальных зависимостей коэффициента оптического отражения в данной работе показывает существенное влияние периодичности системы двумерных слоев плазмонных нановключений AsSb на оптические свойства матрицы среды при том, что размер нановключений был много меньше длины волны света, взаимодействующего со структурой, а их объемная доля - существенно меньше $1 \%$.

Значительный эффект резонансного отражения наблюдался только после формирования системы слоев нановключений металлической фазы вследствие высокотемпературного отжига. С ростом температуры отжига увеличивался размер нановключения и его дипольный момент и, следовательно, увеличивалась степень взаимодействия с внешним электромагнитным полем. Слои $\delta$-Sb сами по себе не вызывали значительного эффекта резонансного отражения света.

На рис. 2 и 3 представлены экспериментальные угловые зависимости резонансной длины волны брэгговской дифракции от угла падения света с $s$ - или $p$-поляризацией для образцов ВР2444 и ST1279, отожженных при температуре $600^{\circ} \mathrm{C}$. Сплошная и штриховая линии показывают результаты расчета по формуле Вульфа-Брэгга (2), в которой эффективные показатель преломления $\left(n_{\mathrm{eff}}\right)$ и пространственный период $\left(d_{\mathrm{eff}}\right)$ структуры использовались в качестве подгоночных параметров. Полученные значения периода и показателя преломления для ВР2444 составили 84.6 нм, 4.46 для $s$-поляризации и 94.7 нм, 3.99 для $p$-поляризации, соответственно. Для образца ST1279 значения параметров равнялись $97.1 \mathrm{Hм}, 3.98$ для $s$-поляризации и $102.6 \mathrm{Hм}$, 3.78 для $p$-поляризации соответственно. Различие эффективных периодов структуры для $s$ - и $p$-поляризаций, повидимому, связано с фазовым сдвигом в распространении света в структурах.

Численное моделирование спектров коэффициента оптического отражения было выполнено с использовани- 
ем метода матриц переноса, обобщенного на периодическую последовательность слоев плазмонных нановключений $\mathrm{AsSb}$ с учетом пространственной геометрии наноструктур ВP2444 и ST1279. Расчеты проводились с учетом экспериментальных данных по оптическому отражению для температуры отжига $700^{\circ} \mathrm{C}$.

Матрица переноса через метаматериал $\mathrm{AsSb}-\mathrm{AlGaAs}$ была сконструирована с учетом резонансных параметров плазмонных возбуждений в слоях нановключений $\mathrm{AsSb}$ в AlGaAs, полученных из экспериментальных данных по оптической экстинкции из работ $[12,13]$. В качестве подгоночных параметров в расчете выступали толщины слоев метаматериала $\mathrm{AsSb}-\mathrm{AlGaAs}$ и спейсеров $\mathrm{AlGaAs,} \mathrm{составлявшие} \mathrm{эффективный} \mathrm{период} \mathrm{исследуе-}$ мой наноструктуры. Дисперсия диэлектрической функции барьеров AlGaAs учитывалась с использованием

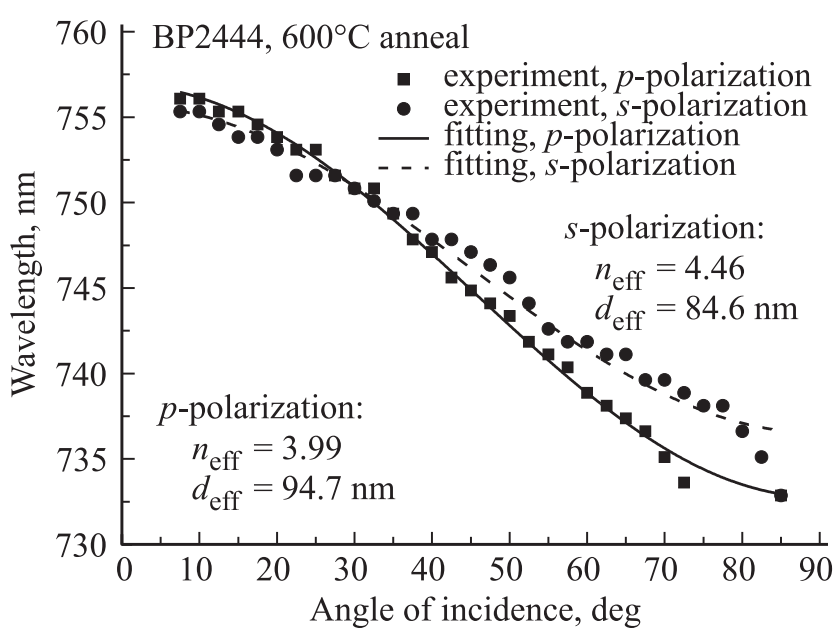

Рис. 2. Зависимости положения брэгговского пика от угла падения света для ВР2444: эксперимент для $p$ - и $s$-поляризации. Линии - расчет по формуле (2), эффективные показатель преломления $\left(n_{\mathrm{eff}}\right)$ и период $\left(d_{\mathrm{eff}}\right)$ указаны.

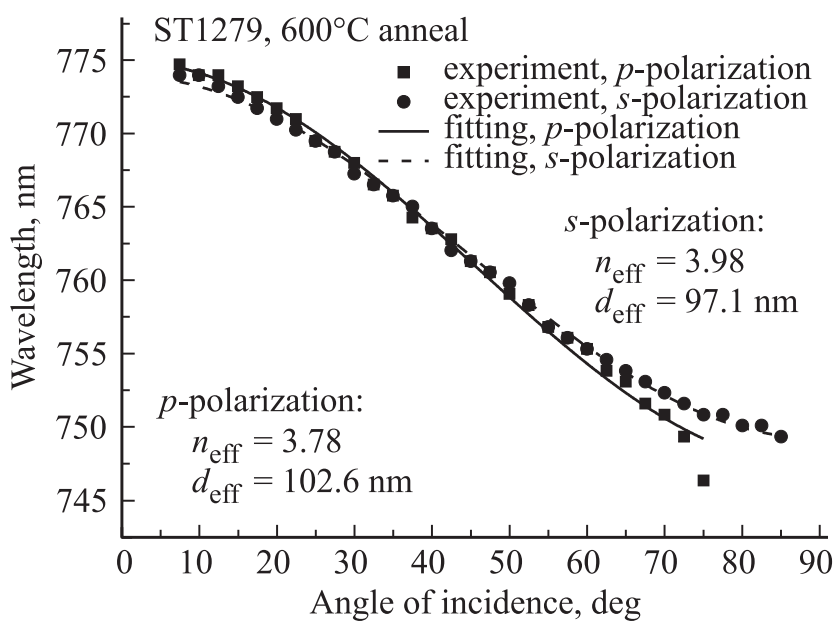

Pис. 3. Зависимости положения брэгговского пика от угла падения света для ST1279: эксперимент для $p$ - и $s$-поляризации. Линии - расчет по формуле (2), эффективные показатель преломления $\left(n_{\mathrm{eff}}\right)$ и период $\left(d_{\mathrm{eff}}\right)$ указаны.

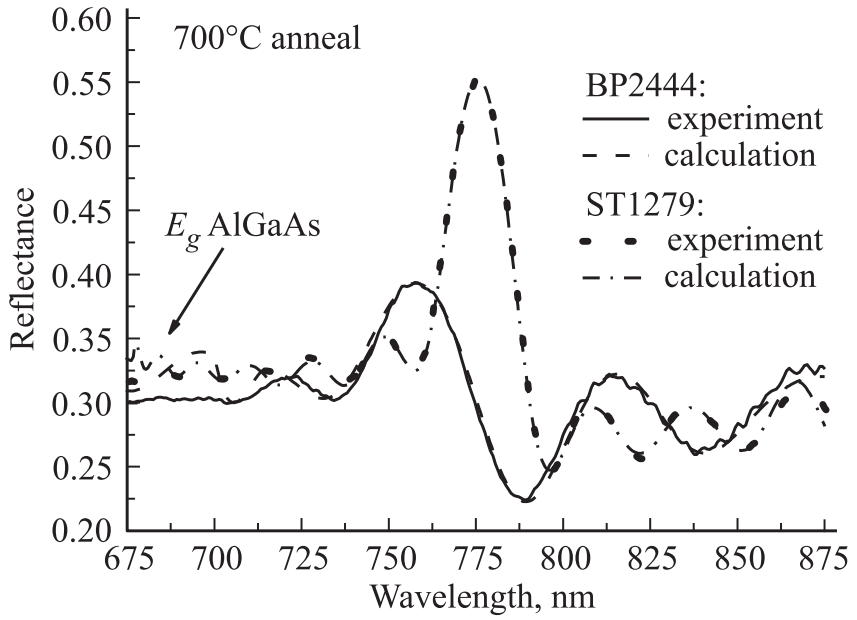

Рис. 4. Сравнение расчетных и экспериментальных спектров коэффициента оптического отражения для образцов ВР2444 и ST1279 при нормальном падении. Температура отжига $700^{\circ} \mathrm{C}$.

соответствующей аппроксимации для тройных сплавов $\mathrm{Al}_{x} \mathrm{Ga}_{1-x} \mathrm{As}$ из работы [16].

Расчетные спектры коэффициента оптического отражения для образцов ВР2444 и ST1279 при нормальном падении представлены на рис. 4 штриховой и штрихпунктирной линиями в сравнении с соответствующими экспериментальными данными. Величины эффективных толщин метаматериала $\mathrm{AsSb}-\mathrm{AlGaAs}$, барьеров $\mathrm{AlGaAs}$ составили 9, 99.5 нм для образца ВР2444, и 9, 102.6 нм для образца ST1279 соответственно. Аналогично экспериментальным расчетные спектры показывают картину резонансной брэгговской дифракции с главным пиком, сопровождающимся боковыми осцилляциями. Расчетные значения положения, амплитуды и полной ширины на половине высоты резонансного пика, а также картина сателлитных осцилляций с хорошей степенью точности совпали с экспериментальными данными.

\section{5. Заключение}

Таким образом, в данной работе исследовалось оптическое отражение метаматериалов на основе полупроводниковой матрицы $\mathrm{AlGaAs}$, содержащей периодические решетки двумерных слоев плазмонных нановключений $\mathrm{AsSb}$. Номинальный период расположения слоев нановключений составлял 100 и 110 нм при числе периодов 12 и 24. Амплитуды основных пиков дифракции достигали 22 и $31 \%$ при том, что объемная доля нановключений $\mathrm{AsSb}$ была существенно меньше 1\%.

В случае падения света под углами, отличными от нормального, длина волны резонансного отражения смещалась в соответствии с законом Вульфа-Брэгга, на основе которого была проведена подгонка эффективных оптических и геометрических параметров для $s$ - и p-поляризаций света.

Численное моделирование спектров коэффициента оптического отражения было выполнено методом матриц 
переноса, обобщенным на случай периодической системы двумерных слоев плазмонных нановключений $\mathrm{AsSb}$ в полупроводниковой матрице AlGaAs. Резонансные свойства слоев $\mathrm{AsSb}-\mathrm{AlGaAs}$ учитывались на основании экспериментальных данных по оптической экстинкции метаматериалов $\mathrm{AsSb}-\mathrm{AlGaAs}$. Расчетные и экспериментальные данные показали хорошее качественное и количественное согласие.

Работа выполнена при частичной поддержке Российского фонда фундаментальных исследований, грант № 14-02-01123 (В.И. Ушанов, В.В. Преображенский, М.А. Путято, Б.Р. Семягин) и программы Президиума РАН „Фундаментальные и прикладные проблемы фотоники и физика новых оптических материалов“ (В.В. Чалдышев).

\section{Список литературы}

[1] M. Kauranen, A.V. Zayats. Nature Photonics, 6, 737 (2012).

[2] K. Yao, Y. Liu. Nanotechnol. Rev., 3 (2), 177 (2014).

[3] M.R. Melloch, N. Otsuka, K. Mahalingam, C.L. Chang, J.M. Woodall, G.D. Pettit, P.D. Kirchner, F. Cardone, A.C. Warren, D.D. Nolte. J. Appl. Phys., 72, 3509 (1992).

[4] D.D. Nolte. J. Appl. Phys., 85, 6259 (1999).

[5] V.V. Chaldyshev. Mater. Sci. Eng. B, 88, 195 (2002).

[6] К. Борен, Д. Хаффмен. Поглощение и рассеяние света малыми частищами (М., Мир, 1986).

[7] М. Борн, Б. Вольф. Основы оптики (М., Наука, 1973).

[8] L.G. Lavrent'eva, M.D. Vilisova, V.V. Preobrazhenski, V.V. Chaldyshev. Crystallography Reports, 41, 1 (2002).

[9] Н.А. Берт, А.И. Вейнгер, М.Д. Вилисова, С.И. Голощапов, И.В. Ивонин, С.В. Козырев, А.Е. Куницын, Л.Г. Лаврентьева, Д.И. Лубышев, В.В. Преображенский, Б.Р. Семягин, В.В. Третьяков, В.В. Чалдышев, М.П. Якубеня. ФТТ, 35, 2609 (1993).

[10] N.A. Bert, V.V. Chaldyshev, A.A. Suvorova, V.V. Preobrazhenskii, M.A. Putyato, B.R. Semyagin, P. Werner. Appl. Phys. Lett., 74, 1588 (1999).

[11] В.В. Чалдышев, М.А. Путято, Б.Р. Семягин, В.В. Преображенский, О.П. Пчеляков, А.В. Хан, В.Г. Канаев, Л.С. Широкова, А.В. Голиков, В.А. Кагадей, Ю.В. Лиленко, Н.В. Карпович. Электронная промышленность, № 1-2, 154 (1998).

[12] В.И. Ушанов, В.В. Чалдышев, Н.Д. Ильинская, Н.М. Лебедева, М.А. Яговкина, В.В. Преображенский, М.А. Путято, Б.Р. Семягин. ФТТ, 56 (10), 1891 (2014).

[13] В.И. Ушанов, В.В. Чалдышев, Н.А. Берт, В.Н. Неведомский, Н.Д. Ильинская, Н.М. Лебедева, В.В. Преображенский, М.А. Путято, Б.Р. Семягин. ФТП, 49 (12), 1635 (2015).

[14] П.В. Лукин, В.В. Чалдышев, В.В. Преображенский, М.А. Путято, Б.Р. Семягин. ФТП, 46, 1314 (2012).

[15] В.И. Ушанов, В.В. Чалдышев, В.В. Преображенский, М.А. Путято, Б.Р. Семягин. ФТП, 47 (8), 1043 (2013).

[16] S. Adachi. J. Appl. Phys., 58, 3 (1985).

\section{Bragg resonance in a system of AsSb plasmon nanoinclusions in AIGaAs}

\author{
V.I. Ushanov ${ }^{+}$, V.V. Chaldyshev ${ }^{+}$, \\ V.V. Preobrazhenskii*, M.A. Putyato*, B.R. Semyagin* \\ + loffe Institute, \\ 194021 St. Petersburg, Russia \\ * Institute of Semiconductor Physics, \\ Siberian Branch of Russian Academy of Sciences, \\ 630090 Novosibirsk, Russia
}

\begin{abstract}
The optical reflection from periodic structures based on a semiconductor AlGaAs matrix containing two-dimensional arrays of plasmonic AsSb nanoinclusions was studied. The number of nanoinclusion layers was 12 or 24 and the nominal spatial periods were 100 or $110 \mathrm{~nm}$, respectively. In the experimental spectra of the optical reflection coefficient at normal incidence we observed a resonant Bragg diffraction with the main peaks at wavelengths of 757 or $775 \mathrm{~nm}(1.64$ or $1.60 \mathrm{eV})$ depending on the spatial period of the nanostructure. The magnitudes of the resonance peaks reached 22 and $31 \%$ for the systems of 12 and 24 layers of $\mathrm{AsSb}-\mathrm{AlGaAs}$, while the volume fraction of nanoinclusions was much less than $1 \%$. In the case of light incident at inclined angles the Bragg diffraction pattern shifted according to the Wulff-Bragg's law. Numerical modeling of optical reflection spectra was performed using the transfer matrix method taking into account spatial structures' geometries and resonant characteristics of the plasmonic AsSb layers.
\end{abstract}

